\title{
Spectral Characteristics of the Microwave Emission by the Spin Hall Nano-Oscillator
}

\author{
R. H. Liu, W. L. Lim, and S. Urazhdin \\ Department of Physics, Emory University, Atlanta, Georgia 30322, USA \\ (Received 2 October 2012; revised manuscript received 26 December 2012; published 1 April 2013)

\begin{abstract}
We utilized microwave spectroscopy to study the magnetization oscillations locally induced in a Permalloy film by a pure spin current, which is generated due to the spin Hall effect in an adjacent Pt layer. The oscillation frequency is lower than the ferromagnetic resonance of Permalloy, indicating that the oscillation forms a self-localized nonpropagating spin-wave soliton. At cryogenic temperatures, the spectral characteristics are remarkably similar to the traditional spin-torque nano-oscillators driven by spin-polarized currents. However, the linewidth of the oscillation increases exponentially with temperature and an additional peak appears in the spectrum below the ferromagnetic resonance, suggesting that the spectral characteristics are determined by interplay between two localized dynamical states.
\end{abstract}

Spin-polarized current is essential for the operation of active spin-based electronic (spintronic) devices [1]. These devices typically utilize two ferromagnetic layers [2]. The current is spin polarized in one layer and injected into another (free) layer, changing the magnetic configuration or causing microwave-frequency precession of the latter due to the spin transfer torque (ST) [3-6]. The ST exerted by each transmitted electron is generally limited by its total spin-angular momentum $\hbar / 2$. As a consequence, device operation requires a relatively large current $I$ that scales with the magnetic moment of the free ferromagnet [3]. On the other hand, thermal stability of nanoscale magnetic devices generally improves with increasing magnetic moment [7]. Because of these opposite trends, it has been challenging to minimize the current required for the operation of spin torque devices while maintaining their stability.

In a recently developed class of spintronic devices, the spin Hall effect (SHE) [8,9] produces a pure spin current controlling the magnetization of ferromagnets [10-14]. The efficiency of SHE devices (the angular momentum transferred to the ferromagnet by each electron transmitted through the device) is not limited by the magnitude of the angular momentum of electron, since each electron can experience multiple scattering between the ferromagnet and the SHE material, transferring angular momentum in each scattering event. Moreover, since no electric current needs to flow through the active magnetic layer, SHE devices can utilize dielectric magnetic materials.

While SHE opens possibilities for new device geometries, it also requires new approaches to signal generation, and a better understanding of the effects of geometry on the dynamical characteristics of magnetic systems. For instance, it has been demonstrated that magnetization oscillations cannot be induced by a uniform spin current applied to a micron-sized ferromagnetic disk because of the nonlinear damping [12].

Auto-oscillation induced by SHE has been recently observed by microfocus Brillouin light spectroscopy
(BLS) in an in-plane point-contact geometry [15]. By locally injecting a spin current into an extended magnetic film, radiative damping of the propagating spin-wave modes was enhanced, suppressing nonlinear processes and enabling auto-oscillation. The oscillation formed a nonpropagating self-localized spin-wave soliton (a spinwave "bullet") at a frequency below the linear spin-wave spectrum of the magnetic material, confirming the earlier theoretical prediction [16]. Reference [15] demonstrated a route for achieving oscillations, but did not show how these oscillations can be converted into microwave signals. The spectroscopic resolution of BLS was also insufficient to determine the spectral properties of the oscillation.

Here, we demonstrate coherent microwave generation due to the SHE in a device that utilizes local current injection to suppress nonlinear damping, and anisotropic magnetoresistance (AMR) of the magnetic layer to convert the oscillations into a microwave signal. Our spectroscopic measurements directly demonstrate the coherent singlemode nature of the excitation and confirm its relation to the spin-wave spectrum observed in Ref. [15]. Furthermore, we show that while at low temperatures the linewidth is comparable to other magnetic nanooscillators, at higher temperatures it significantly increases and an additional peak appears in the spectrum, suggesting a greater complexity of the dynamical states induced by spin current than previously theoretically predicted [16].

Figure 1(a) shows a scanning electron microscopy (SEM) image of our test device and the experimental layout. The device is comprised of a $4-\mu \mathrm{m} \mathrm{Py}(5) / \mathrm{Pt}(4)$ bilayer disk and two pointed $\mathrm{Au}(100)$ electrodes on top. Here, Py is Permalloy $\left(\mathrm{Ni}_{80} \mathrm{Fe}_{20}\right)$, and thicknesses are in nanometers. The device was fabricated by a combination of sputtering and e-beam lithography. The separation between the endpoints of the Au electrodes was $70 \mathrm{~nm}$. By applying a voltage between the Au electrodes, an inplane electrical current localized mostly in the gap between electrodes was induced in the $\mathrm{Py} / \mathrm{Pt}$ bilayer. This current 

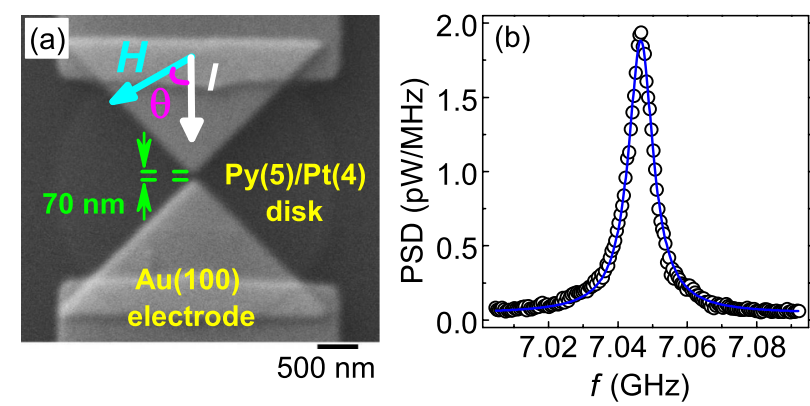

FIG. 1 (color online). (a) Scanning electron micrograph of the test device. (b) Symbols: power spectral density (PSD) of the microwave signal emitted by the device at $H=700 \mathrm{Oe}, I=$ $20 \mathrm{~mA}, T=6 \mathrm{~K}$, at an angle $\theta=60^{\circ}$ between the direction of the field and the current flow. The curve is the result of fitting by the Lorentzian function.

generated a pure spin current flowing towards the Py layer due to the SHE in Pt [8,9], resulting in the oscillation of the Py magnetization $\mathbf{M}$.

To enable microwave generation due to this oscillation, we utilized the dependence of the device resistance $R$ on the angle $\theta$ between $\mathbf{M}$ and the direction of current $I$. Magnetometry measurements showed that the coercivity of the Py film did not exceed 5 Oe, significantly smaller than the magnitude of the applied in-plane magnetic field $H \geq 180$ Oe [17]. Therefore, the equilibrium orientation of $\mathbf{M}$ was parallel to the field $\mathbf{H}$. The sinusoidal dependence of $R$ on the orientation of $\mathbf{H}$ with a period of $180^{\circ}$ was consistent with the anisotropic magnetoresistance (AMR) of Py [18]. The relative magnetoresistance was $\Delta R / R=$ $\left[R\left(0^{\circ}\right)-R\left(90^{\circ}\right)\right] / R\left(90^{\circ}\right)=0.125 \%$ at $\mathrm{RT}$, smaller than in stand-alone Py films due to the shunting by Pt.

The effects of ST in SHE devices $\mathbf{M}$ are most significant when the direction of $\mathbf{M}$ is orthogonal to $I[12,14]$, corresponding to the minimum of AMR. Therefore, a microwave signal at the oscillation frequency cannot be produced in this configuration. Indeed, we observed oscillations only at intermediate angles $\theta$ between $30^{\circ}$ and $85^{\circ}$, as illustrated in Fig. 1(b) for $\theta=60^{\circ}$. The spectrum can be fitted by the Lorentzian function with a full width at half maximum (FWHM) of $5 \mathrm{MHz}$ at $T=6 \mathrm{~K}$ [solid curve in Fig. 1(b)].

Figure 2 shows the dependence of the generation characteristics on $I$ at $T=120 \mathrm{~K}$. Above the onset at $I=$ $14 \mathrm{~mA}$, the intensity of the oscillation peak increased to a maximum power spectral density (PSD) of $110 \mathrm{pW} / \mathrm{MHz}$ at $I=19 \mathrm{~mA}$ and the frequency slightly increased, while the linewidth decreased to a minimum value of $10 \mathrm{MHz}$ at $I=19 \mathrm{~mA}$. At $I>19 \mathrm{~mA}$, the peak broadened and decreased in amplitude while shifting to lower frequencies. This evolution of the oscillation characteristics is remarkably similar to the traditional spinvalve nano-oscillators both in the nanopillar [19] and point contact geometries [20,21]. It is consistent with the theory of nonlinear oscillators, which predicts that the thermal
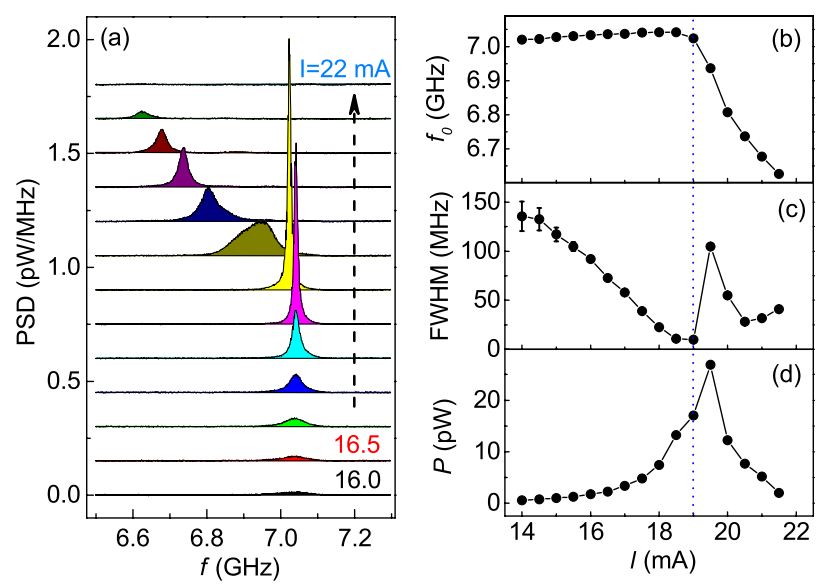

FIG. 2 (color online). Dependence of the microwave generation characteristics on current, at $H=700 \mathrm{Oe}, \theta=60^{\circ}$, $T=120 \mathrm{~K}$. (a) Generation spectra at $16 \mathrm{~mA}<I<22 \mathrm{~mA}$ varied in $0.5 \mathrm{~mA}$ increments. (b)-(d) Dependence of the central generation frequency (b), FWHM (c), and integral intensity (d) on current. The central frequency and the linewidth were determined by fitting the power spectra with the Lorentzian function. Dotted vertical line marks the current $I_{p}$ defined in text.

linewidth decreases with increasing oscillation power and increases with increasing nonlinearity $[22,23]$. In particular, a significant broadening of the oscillation peak at $I>19 \mathrm{~mA}$ is correlated with a strong oscillation redshift.

The peak discussed above was the only spectral feature observed at low temperatures. However, at higher temperatures and large currents, another peak appeared at higher frequency while the low-frequency peak became gradually suppressed [see Fig. 3(a)]. The higher-frequency peak exhibited a larger linewidth and persisted to room temperature. Two spectral peaks have been previously simultaneously observed in point-contact magnetic nanooscillators [24]. The low-frequency peak was identified as the self-localized, spin-wave "bullet" mode, while the other peak was identified as a propagating mode within the linear spin-wave spectrum.

To establish the relation of the observed oscillation peaks to the spin-wave spectrum of the Py film, we determined the ferromagnetic resonance (FMR) frequency $f_{\mathrm{FMR}}$ by the spin torque-driven FMR (ST-FMR) technique [25]. An ac current $I_{a c}$ was applied at a microwave frequency $f_{\text {ext }}$, causing magnetization oscillation due to a combination of the Oersted field and ST. The resulting periodic variation of resistance due to AMR mixed with the ac current, producing a peak of dc voltage across the sample at the value of $H$ corresponding to $f_{\mathrm{FMR}}=f_{\text {ext }}$.

We obtained good fits to the ST-FMR peaks with a sum of symmetric and antisymmetric Lorentizan contributions [see inset in Fig. 3(b)], consistent with the previous studies $[11,26]$. The dependence of $f_{\mathrm{FMR}}$ on $H$ agreed well with the Kittel formula [solid curve in Fig. 3(b)]. The value $M=827 \mathrm{G}$ of Py magnetization obtained from the best fit 

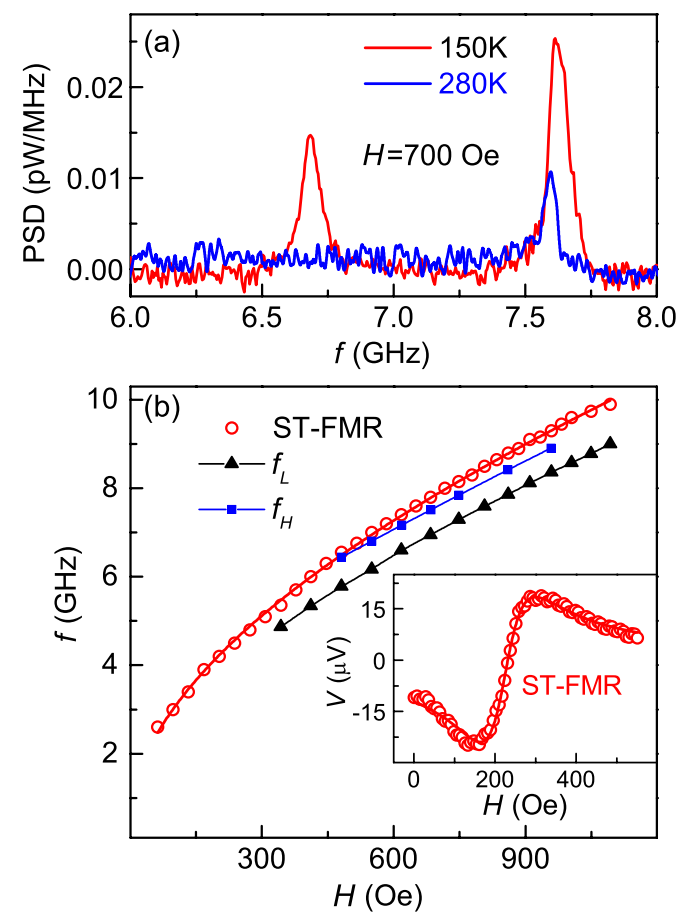

FIG. 3 (color online). Relation of the oscillation peaks to the ferromagnetic resonance (FMR) of the Py film. (a) Microwave generation spectra at $T=150 \mathrm{~K}, I=25 \mathrm{~mA}$ and $T=280 \mathrm{~K}$, $I=24 \mathrm{~mA}$. (b) The ST-FMR frequency (circles), the maximum frequency of the low-frequency peak (triangles) and the highfrequency peak (squares) vs $H$. The solid curve is the result of fitting the FMR data with the Kittel formula $f=$ $\gamma \sqrt{H(H+4 \pi M)}$, where $M=827 \mathrm{G}$ is the best-fit value of the Py magnetization, and $\gamma=2.8 \mathrm{MHz} / \mathrm{Oe}$ is the gyromagnetic ratio. Inset: the ST-FMR voltage vs field $H$ obtained with an ac current of $2.5 \mathrm{~mA} \mathrm{rms}$ at frequency $f_{\text {ext }}=4.5 \mathrm{GHz}$. The curve is the best fit with a sum of a symmetric and an antisymmetric Lorentzian. $f_{\mathrm{FMR}}$ and $f_{L}$ are determined at $T=6 \mathrm{~K}$, and $f_{H}$ is determined at $T=150 \mathrm{~K}$, since this peak appears only at higher temperatures.

was consistent with our magnetometry measurements [17] and the published data for Py $[11,26]$.

At $T=6 \mathrm{~K}$ and $H=1.1 \mathrm{kOe}$, the maximum frequency $f_{L}$ of the low-frequency peak was $0.9 \mathrm{GHz}$ below $f_{\mathrm{FMR}}$ [Fig. 3(b)]. The difference between the two frequencies decreased with decreasing $H$ to $0.5 \mathrm{GHz}$ at $H=340 \mathrm{Oe}$. This relationship between $f_{L}$ and $f_{\mathrm{FMR}}$ was preserved at higher temperatures, confirming the findings of Ref. [15] and the prediction of Ref. [16] that the auto-oscillation mode does not belong to the linear spin-wave spectrum, but instead forms a nonpropagating, self-localized "bullet" mode at a frequency below the spectrum.

The higher-frequency peak appeared only at higher temperatures and large currents. Its frequency $f_{H}$ was very close to, but always below $f_{\mathrm{FMR}}$, suggesting that this is also a nonpropagating localized mode. Similar characteristics were exhibited by another studied device with a larger gap between the electrodes [17]. These results indicate that the dynamical states induced by spin current are more complicated than envisioned in the original theoretical predictions [16]. We note that the Oersted field of the current in our devices opposes external field, reducing the frequency of the oscillation. It is possible that the high-frequency mode can become propagating if the Oersted field is reversed by utilizing a different spin Hall material such as Ta, which is characterized by the opposite sign of the spin Hall angle [27].

Analysis of the temperature dependence of the oscillation provided further evidence for the bimodal dynamics induced by spin current [Fig. 4]. To take into account Joule heating, we first measured the dependence of the device resistance on current at different values of $T$, as illustrated by curves in Fig. 4(a). The dependence of resistance on current is approximately linear at low temperatures, and quadratic at high temperatures, as expected for Joule heating effects [28]. By comparing these dependencies to $R$ vs $T$ [red curve and top scale in Fig. 4(a)], we obtained the dependence of the actual device temperature $T_{a}$ on $I$ at a given $T$.
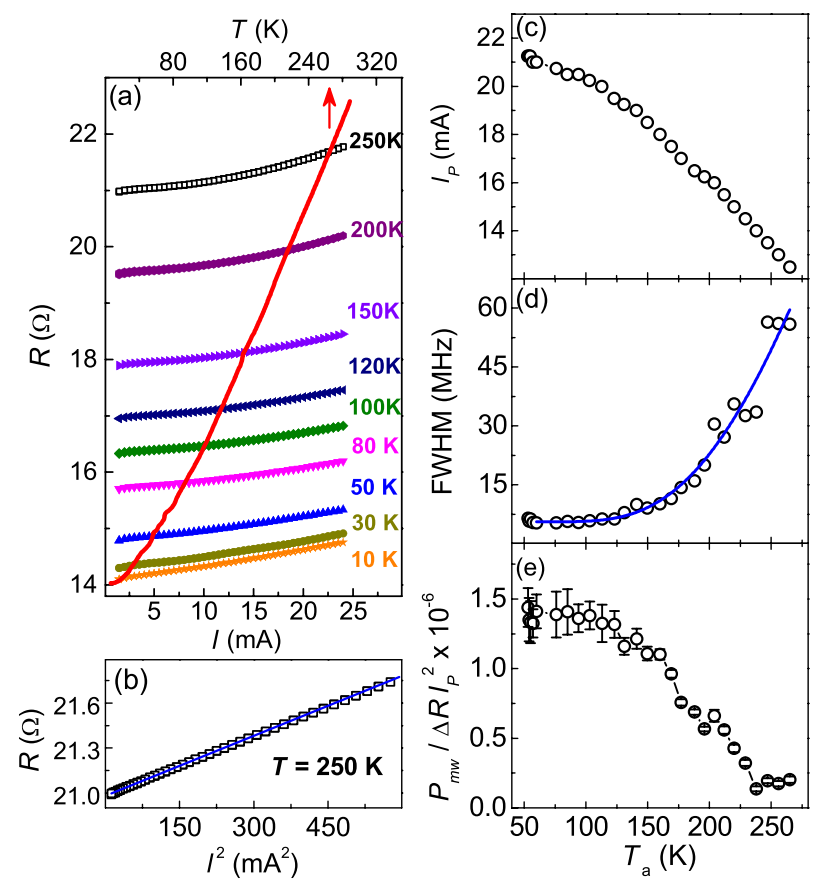

FIG. 4 (color online). Effects of temperature on the spectral characteristics of the low-frequency mode. (a) $R$ vs $I$ at different substrate temperatures, as labeled (bottom axis), and $R$ vs $T$ measured with a small ac current (top axis). (b) $R$ vs $I^{2}$ at $250 \mathrm{~K}$. Solid line is a linear fit to the data. (c) The dependence of the current value $I_{P}$ corresponding to the highest peak PSD on the calculated temperature $T_{a}$ of the active device area. (d) Dependence of the linewidth at $I_{P}$ on $T_{a}$. Solid curve is the result of fitting by an exponential dependence $A+$ $B \exp \left(-E_{b} / k_{B} T\right)$ with $E_{b}=90 \mathrm{meV}$. (e) $P_{m w} / \Delta R I_{P}^{2}$ vs $T_{a}$, where $P_{m w}$ is the integrated microwave power under the lowfrequency peak. 
We now analyze the dependence of the oscillation characteristics on $T_{a}$. The precise value of the oscillation threshold current and the oscillation characteristics at this current are difficult to determine due to the thermal noise. To avoid this difficulty, we define temperature-dependent current $I_{P}$ corresponding to the highest peak PSD $\left[I_{P}=19 \mathrm{~mA}\right.$ at $T=120 \mathrm{~K}$ in Fig. 2(a)]. This current value is marked by a dotted vertical line in Figs. 2(b)-2(d). We note that the oscillation frequency reaches a maximum at a current value $I=18.5 \mathrm{~mA}$ slightly smaller than $I_{P}$ [Fig. 2(b)], while the total generated power exhibits a maximum at a slightly larger current $I=19.5 \mathrm{~mA}$ [Fig. 2(d)].

Figure 4(c) shows that $I_{P}$ decreases from $21.2 \mathrm{~mA}$ at $T_{a}=50 \mathrm{~K}$ to $12.5 \mathrm{~mA}$ at $T_{a}=265 \mathrm{~K}$. We observed a similar temperature dependence for the threshold current. The apparent increase of the excitation efficiency at higher temperatures may be caused by a decrease of the Py magnetization $\mathbf{M}$, an increase of the spin-current generated by SHE, and/or a decrease of the dynamical damping. Our measurements of ST-FMR did not show a significant variation of $f_{\mathrm{FMR}}$ with $T$, indicating that $\mathbf{M}$ does not significantly vary over the studied temperature range. We also expect that the damping of oscillation increases with increasing $T$, due to the larger contribution of nonlinear interaction of oscillation with thermal spin-waves. Therefore, the observed behaviors are likely associated with the increase of spin-current generated by SHE, warranting further studies of the temperature dependence of this effect.

We now focus our analysis on the spectral characteristics of the low-frequency peak at $I=I_{P}$. Figure 4(d) shows the dependence of the linewidth on $T_{a}$ at $I=I_{P}$. It remains approximately constant at $T_{a}<150 \mathrm{~K}$, but rapidly increases at larger $T_{a}$. This dependence is qualitatively different from the thermal broadening of single-mode oscillation [7,23], indicating a significant effect of the additional higher-frequency mode that appears at high temperatures. Indeed, exponential dependence of linewidth on temperature in some spin-valve nano-oscillators has been identified with thermally activated transitions between different dynamical modes [29,30]. Based on the two-state fluctuation model, we can fit the FWHM with $A+B \exp \left(-E_{b} / k_{B} T\right)$, with the best-fit value of $90 \mathrm{meV}$ for the energy barrier $E_{b}$ between the two states [curve in Fig. 4(d)].

Analysis of the microwave generation power provides additional evidence for the bimodal effects. Figure 4(e) shows the temperature dependence of the integrated power $P_{m w}$ under the low-frequency peak at $I=I_{P}$, normalized by $\Delta R I_{P}^{2}$ to eliminate the effects of the variations of AMR and applied current on the microwave generation. This normalized quantity characterizes the average oscillation amplitude of the active device area. The amplitude remains approximately constant at $T_{a}<120 \mathrm{~K}$, but decreases by almost an order of magnitude at $T_{a}=240 \mathrm{~K}$. These behaviors are correlated with the variations of the linewidth [Fig. 4(e)], consistent with the increasing dwell time of the higherfrequency mode relative to the low-frequency one.

To summarize, we have demonstrated emission of coherent microwaves due to the magnetization oscillation induced by a local pure spin current in a Py/Pt bilayer, which is converted into a microwave signal due to the anisotropic magnetoresistance of Py. The oscillation frequency was always lower than the frequency of the ferromagnetic resonance, confirming that the oscillation mode is a self-localized standing spin-wave. The dependence of the oscillation characteristics on current was remarkably similar to the spin-valve nano-oscillators. However, we observe a strong increase of the oscillation linewidth with temperature correlated with the emergence of an additional higher-frequency oscillation mode. These results provide insight into the complexity of the dynamical magnetization states induced by the local spin currents, suggesting that nonlinear dynamical phenomena in nanoscale magnetic systems are still not well understood.

After the manuscript submission, we learned about an alternative solution for the electronic detection of magnetization oscillation in a spin Hall device utilizing a threeelectrode structure incorporating a magnetic tunnel junction [31].

We thank Rongying Jin for the magnetic characterization. This work was supported by the NSF Grants No. ECCS-1218419 and No. DMR-1218414.

[1] G. A. Prinz, Science 282, 1660 (1998).

[2] J. A. Katine, F. J. Albert, R. A. Buhrman, E. B. Myers, and D. C. Ralph, Phys. Rev. Lett. 84, 3149 (2000).

[3] J. C. Slonczewski, J. Magn. Magn. Mater. 159, L1 (1996); J. Magn. Magn. Mater. 195, L261 (1999).

[4] L. Berger, Phys. Rev. B 54, 9353 (1996); J. Appl. Phys. 90, 4632 (2001).

[5] M. Tsoi, A. G. M. Jansen, J. Bass, W.-C. Chiang, V. Tsoi, and P. Wyder, Nature (London) 406, 46 (2000).

[6] S. I. Kiselev, J. C. Sankey, I. N. Krivorotov, N. C. Emley, R. J. Schoelkopf, R. A. Buhrman, and D. C. Ralph, Nature (London) 425, 380 (2003).

[7] T. Silva and M. Keller, IEEE Trans. Magn. 46, 3555 (2010).

[8] M. I. Dyakonov and V. I. Perel, Sov. Phys. JETP Lett. 13, 467 (1971).

[9] J.E. Hirsch, Phys. Rev. Lett. 83, 1834 (1999).

[10] K. Ando, S. Takahashi, K. Harii, K. Sasage, J. Ieda, S. Maekawa, and E. Saitoh, Phys. Rev. Lett. 101, 036601 (2008).

[11] L. Liu, T. Moriyama, D. C. Ralph, and R. A. Buhrman, Phys. Rev. Lett. 106, 036601 (2011).

[12] V. E. Demidov, S. Urazhdin, E. R. J. Edwards, M. D. Stiles, R. D. McMichael, and S. O. Demokritov, Phys. Rev. Lett. 107, 107204 (2011).

[13] Z. H. Wang, Y. Y. Sun, M.Z. Wu, V. Tiberkevich, and A. Slavin, Phys. Rev. Lett. 107, 146602 (2011).

[14] L. Liu, O. J. Lee, T. J. Gudmundsen, D. C. Ralph, and R. A. Buhrman, Phys. Rev. Lett. 109, 096602 (2012). 
[15] V. E. Demidov, S. Urazhdin, H. Ulrichs, V. Tiberkevich, A. Slavin, D. Baither, G. Schmitz, and S. O. Demokritov, Nat. Mater. 11, 1028 (2012).

[16] A. Slavin and V. Tiberkevich, Phys. Rev. Lett. 95, 237201 (2005).

[17] See Supplemental Material at http://link.aps.org/ supplemental/10.1103/PhysRevLett.110.147601 for the magnetic properties of $\mathrm{Py} / \mathrm{Pt}$ films and spectral characteristics of another spin Hall oscillator.

[18] R. C. O'Handley, Modern Magnetic Materials: Principles and Applications (Wiley, New York, 1999).

[19] Q. Mistral, J.-V. Kim, T. Devolder, P. Crozat, and C. Chappert, J. A. Katine, M. J. Carey, and K. Ito, Appl. Phys. Lett. 88, 192507 (2006).

[20] P. Tabor, V. Tiberkevich, A. Slavin, and S. Urazhdin, Phys. Rev. B 82, 020407 (2010).

[21] S. Urazhdin, V. Tiberkevich, and A. Slavin, Phys. Rev. Lett. 105, 237204 (2010).

[22] J.-V. Kim, Q. Mistral, C. Chappert, V. S. Tiberkevich, and A. N. Slavin, Phys. Rev. Lett. 100, 167201 (2008).

[23] A. Slavin and V. Tiberkevich, IEEE Trans. Magn. 44, 1916 (2008); 45, 1875 (2009).
[24] S. Bonetti, V. Tiberkevich, G. Consolo, G. Finocchio, P. Muduli, F. Mancoff, A. Slavin, and J. Akerman, Phys. Rev. Lett. 105, 217204 (2010).

[25] G. D. Fuchs, J. C. Sankey, V.S. Pribiag, L. Qian, P. M. Braganca, A. G. F. Garcia, E. M. Ryan, Z.-P. Li, O. Ozatay, D. C. Ralph, and R. A. Buhrman, Appl. Phys. Lett. 91, 062507 (2007).

[26] O. Mosendz, J. E. Pearson, F. Y. Fradin, G. E. W. Bauer, S. D. Bader, and A. Hoffmann, Phys. Rev. Lett. 104, 046601 (2010).

[27] L. Liu, C.-F. Pai, Y. Li, H. W. Tseng, D. C. Ralph, and R. A. Buhrman, Science 336, 555 (2012).

[28] R. Holm, Electric Contacts (Springer-Verlag, Berlin, 1967), p. 63.

[29] J. C. Sankey, I. N. Krivorotov, S. I. Kiselev, P. M. Braganca, N. C. Emley, R. A. Buhrman, and D. C. Ralph, Phys. Rev. B 72, 224427 (2005).

[30] M. L. Schneider, W.H. Rippard, M.R. Pufall, T. Cecil, T. J. Silva, and S.E. Russek, Phys. Rev. B 80, 144412 (2009).

[31] L. Liu, C.-F. Pai, D. C. Ralph, and R. A. Buhrman, Phys. Rev. Lett. 109, 186602 (2012). 